\title{
A New Algorithm for Solving Linear Bottleneck Assignment Problem
}

\author{
Pramendra Singh Pundir ${ }^{1}$, Sandeep Kumar Porwal ${ }^{2}$ and Brijesh P. Singh ${ }^{3}$ \\ ${ }^{I}$ Department of Statistics, University of Allahabad, Allahabad \\ Banaras Hindu University, Varanasi, INDIA \\ ${ }^{2}$ DST-CIMS, Banaras Hindu University, Varanasi, INDIA. \\ ${ }^{3}$ Faculty of Commerce and DST-CIMS, BHU, Varanais, INDIA \\ E-mail:brijesh@bhu.ac.in
}

\begin{abstract}
Linear bottleneck assignment problems have wide range of applications in emergency situation like call for fire fighters, ambulances etc. This paper presents a new simple but faster algorithm for solving linear bottleneck assignment problems, so that in such emergent situation efficient and faster decision taking could be make possible. Some examples are also discussed to explain the procedure.
\end{abstract}

Keywords: Linear bottleneck assignment problems, improved solution, optimal value.

\section{INTRODUCTION}

Around 60 years back, in 1955 and 1956 Harold (1955) published two famous articles presenting the Hungarian algorithm, the first polynomial-time method for the assignment problem. This historic result allowed the researchers for the first time an easy solution of real-world instances that no computer on earth could then handle. Since then the assignment problem has attracted hundreds of researchers, accompanying and sometimes anticipating the development of combinatorial optimization. Burkard et al. (2009) had given a comprehensive view of this huge area, starting from the conceptual foundations laid down since the 1920s by the studies on matching problems, and examining in detail theoretical, algorithmic, and practical developments of the various assignment problems.

Linear bottleneck assignment problems (LBAP) were introduced by Fulkerson et al. (1953) in connection with assigning jobs to parallel machines so as to minimize the latest completion time. In this paper, an improved algorithm for solving LBAP is discussed with examples.

\section{THE ALGORITHM}

For the given cost matrix $\mathrm{C}=\left[\mathrm{c}_{\mathrm{ij}}\right]$

Step 1: Define the $\mathrm{c}^{*}=\operatorname{Max}\left(\operatorname{Min}_{\mathrm{i}} \mathrm{c}_{\mathrm{ij}}, \operatorname{Min}_{\mathrm{j}} \mathrm{c}_{\mathrm{ij}}\right)$

Step 2: Replace all those entries which are greater than $\mathrm{c}^{*}$ by $\infty$.
Step 3: Obtain efficient solution by using Hungarian method. If solution exist then $\mathrm{c}^{*}$ is optimal value for LBAP.

Otherwise,

Step 4: Write down all the entries of the cost matrix $\mathrm{C}=\left[\mathrm{c}_{\mathrm{ij}}\right]$ in ascending order and take next ordered value larger than $\mathrm{c}^{*}$, as improved $\mathrm{c}^{*}$. Now, repeat step 2 and 3 till the solution is obtained.

\section{EXAMPLES}

Here we consider, two examples from Burkard et al. (2009).

Example 1: Consider an LBAP with cost matrix

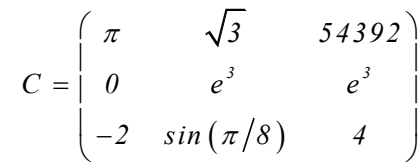

Solution:

Step 1: $\mathrm{c}^{*}=\operatorname{Max}(-2, \sin (\pi / 8), 4, \sqrt{3}, 0,-2)=4$

Step 2: Using Hungarian method we get the solution as $(2,1,3)$.

Example 2: Consider an LBAP with cost matrix

$$
C=\left(\begin{array}{llll}
8 & 2 & 3 & 3 \\
2 & 7 & 5 & 8 \\
0 & 9 & 8 & 4 \\
2 & 5 & 6 & 3
\end{array}\right)
$$


Solution:

Step 1: $\mathrm{c}^{*}=\operatorname{Max}(2,2,0,2,0,2,3,3)=3$

Step 2: Using Hungarian method we could not get the solution.

Step 3: Ordered arrangement of the $\mathrm{c}_{\mathrm{ij}} \mathrm{s}$ is $0,2,2,2$, $3,3,3,4,5,5,6,7,8,8,8,9$. Now, take $\mathrm{c}^{*}=4$.

Step 4: Again by using Hungarian method we could not get the solution.

Step 5: Now take $\mathrm{c}^{*}=5$.

Step 6: Using Hungarian method we get the solution as $(2,3,1,4)$.

\section{CONCLUSION}

LBAP has wide variety of applications in emergency situation like call for fire fighters or ambulances or various situations during war etc. This algorithm not only provides a simpler tool but also a faster tool to decision makers as it gives the optimal answer in either less iterations (i.e. number of times $c^{*}$ is defined) or at the most in as many iterations taken by any other popular method like threshold algorithm, augmenting path methods etc.

\section{REFERENCES}

Burkard, R., Dell'Amico, M., Martello, S. 2009. Assignment Problems. SIAM.

Fulkerson, D.R., Glicksberg, I., Gross, O. 1953. A production line assignment problem. Tech. Rep. RM-1102, The Rand Corporation, Santa Monica, CA.

Kuhn, H.W. 1955. The Hungarian method for the assignment problem. Naval Res. Log. Quart. 2: 83-97.

Kuhn, H.W. 1956. Variants of the Hungarian method for the assignment problem. Naval Res. Log. Quart. 3: 253-258. 